TRANSACTIONS OF THE

AMERICAN MATHEMATICAL SOCIETY

Volume 196, 1974

\title{
NEW CRITERIA FOR FREENESS IN ABELIAN GROUPS. II(1)
}

\author{
BY
}

PAUL HILL

ABSTRACT. A new criterion is established for an abelian group to be free. The criterion is in terms of an ascending chain of free subgroups and is dependent upon a new class of torsion-free groups. The result leads to the construction, for each positive integer $n$, of a group $G_{n}$ of cardinality $X_{n}$ that is not free but is $x_{n}$-free. A conjecture in infinitary logic concerning free abelian groups is also verified.

1. Introduction. Recall that for any uncountable cardinal number $x_{a}$, an abelian group is called $x_{\alpha}$-free if each subgroup of cardinality less that $x_{a}$ is free. Problem 10 in [3] asks for a determination of those ordinals $a$ such that there exist $x_{a}$-free abelian groups that are not $x_{a+1}$-free. Shortening "abelian group" to "group", we remark that examples are in abundance of $x_{1}$-free groups that are not $x_{2}$-free. Indeed, such examples exist within a countable product of integers. Recently, the author has constructed groups in [5] that are $x_{2}$ free but not $x_{3}$-free. In this connection, P. Griffith [4] has just shown the existence, for each positive integer $n$, of a group $G_{n}$ that is $\aleph_{n}$-free but not free. However, there is one aspect of Griffith's construction that leaves room for some improvement. The construction does not determine the minimal cardinality of $G_{n}$, but exhibits only an upper bound that tends to be relatively large. It would be more useful, for most purposes, if it were known that $G_{n}$ could be chosen to have cardinality $x_{n}$, which is the lower bound. In this paper, we are able to construct such a group $G_{n}$. Thus we provide a partial answer to Problem 10 in [3]. Our construction is based on a strong uniqueness theorem established herein, and it also yields the proof of a conjecture made in [6].

2. The main theorem. By a smooth chain of groups, we shall mean an ascending chain

$$
G_{0} \subseteq G_{1} \subseteq \cdots \subseteq G_{a} \subseteq \cdots, \quad a<\mu,
$$

indexed by an ordinal $\mu$, with the property that $G_{\beta}=\bigcup_{a<\beta} G_{a}$ whenever $\beta$ is a

Received by the editors June 20, 1973.

AMS (MOS) subject classifications (1970), Primary 20K20; Secondary 20E15.

Key words and phrases. Smooth chain of free groups, $\boldsymbol{x}_{n}$-free, $\boldsymbol{Z}_{n}$-free groups, nonfree $X_{n}$-free groups, equivalence in $L_{\infty \mathrm{OK}}{ }^{\circ}$

(1) This research was sponsored, in part, by NSF Grant GP-29025. 
limit ordinal less than $\mu$. Let $\omega_{n}$ denote the first ordinal having cardinality $x_{n}$. For each nonnegative integer $n$, a class $\mathcal{F}_{n}$ of torsion-free groups is defined as follows. The class $\mathfrak{F}_{0}$ consists of all countable torsion-free groups. Inductively, we define $\mathcal{F}_{n}$ to be the class of torsion-free groups $G$ of cardinality not exceeding $x_{n}$ that can be represented as the union of a smooth chain $G_{0} \subseteq G_{1} \subseteq \cdots \subseteq G_{a} \subseteq$ $\cdots, a<\mu$, of free groups $G_{a}$, where $\mu \leq \omega_{n}$ and $G_{a+1} / G_{\alpha} \in \mathcal{F}_{n-1} \cdot$

We begin with an elementary but useful result.

Theorem 1. Let $H$ be a subgroup of $G$. If $G \in \mathcal{F}_{n}$, then $H \in \mathcal{F}_{n}$.

Proof. The proof is by induction on $n$, and the theorem is trivial if $n=0$. Let $G$ be the union of the smooth chain $G_{0} \subseteq G_{1} \subseteq \cdots \subseteq G_{a} \subseteq \cdots, a<\mu$, of free groups $G_{a}$, where $\mu \leq \omega_{n}$ and $G_{a+1} / G_{a} \in \mathcal{F}_{n-1}$. For each $a<\mu$, define $H_{a}=$ $H \cap G_{a}$. Obviously, $H_{a}$ is free and $H$ is the union of the smooth chain

$$
H_{0} \subseteq H_{1} \subseteq \cdots \subseteq H_{a} \subseteq \cdots, \quad a<\mu,
$$

of free groups. Since $H_{a+1} / H_{\alpha}=H_{a+1} / H_{a+1} \cap G_{a}$ and the latter is isomorphic to a subgroup of $G_{a+1} / G_{a}$, we conclude by the induction hypothesis that $H_{a+1} / H_{a} \epsilon$ $\mathcal{F}_{n-1}$. Hence $H \in \mathcal{F}_{n}$.

Although we are not prepared by any means for its proof, we shall now state the main theorem.

Main Theorem. If $G \in \mathcal{F}_{n}$, then $G$ is $x_{n}$-free for every positive integer $n$.

In order to prove our main theorem, the following results are needed.

Lemma. If $G \in \mathcal{F}_{n}$ for a positive integer $n$, then $G$ can be represented as the union of a smooth chain $G_{0} \subseteq G_{1} \subseteq \cdots \subseteq G_{a} \subseteq \cdots, a<\omega_{n}$, of free groups $G_{a}$ such that $\left|G_{a}\right| \leq{\aleph_{n-1}}$ and $G_{\beta} / G_{a} \in \mathcal{F}_{n-1}$ if $a<\beta<\omega_{n}$.

Proposition. Let $A \longrightarrow G \rightarrow B$ be a short exact sequence. If $A$ and $B$ are in $\mathcal{F}_{n}$, then $G \in \mathcal{F}_{n}$.

Corollary. If $G_{0} \subseteq G_{1} \subseteq \cdots \subseteq G_{a} \subseteq \cdots, a<\mu$, is a smooth chain of groups $G_{a} \in \mathcal{F}_{n}$ with $G_{a+1} / G_{a} \in \mathcal{F}_{n}$ and $|\mu| \leq x_{n}$, then $G=\bigcup_{a<\mu} G_{a}$ belongs to $\mathcal{F}_{n}$.

For a fixed nonnegative integer $n$, the symbols $T(n), L(n), P(n)$ and $C(n)$ will denote, respectively, main theorem, lemma, proposition and corollary restricted to the given integer $n$. Therefore, for example, the symbol

$$
P(n-1)+L(n) \Rightarrow P(n)
$$

means that if the proposition is true for $n-1$ and the lemma is true for $n$, then the proposition must be true for $n$. $\Lambda s$ might be expected, there are a number of 
implications among the results that we have stated above and now have the burden of proving. The next four sections are devoted to verifying some of the most important of these implications. Fortunately, the implications culminate in the proof of the main theorem.

3. $P(n-1)+L(n) \Rightarrow P(n)$. Let $A \longrightarrow G \rightarrow B$ be exact where $A$ and $B$ are in $\mathcal{F}_{n}$. By $L(n), A$ can be represented as the union of a smooth chain

$$
A_{0} \subseteq A_{1} \subseteq \cdots \subseteq A_{a} \subseteq \cdots, \quad \alpha<\omega_{n},
$$

of free groups $A_{a}$ such that $\left|A_{a}\right| \leq x_{n-1}$ and $A_{\beta} / A_{a} \in \mathcal{F}_{n-1}$ if $\alpha<\beta<\omega_{n}$. Likewise, $B$ has a similar representation as the union of

$$
B_{0} \subseteq B_{1} \subseteq \cdots \subseteq B_{\alpha} \subseteq \cdots, \quad \alpha<\omega_{n} .
$$

It should be evident that $G$ is the union of a smooth chain

$$
G_{0} \subseteq G_{1} \subseteq \cdots \subseteq G_{a} \subseteq \cdots, \quad a<\omega_{n},
$$

of subgroups $G_{\alpha}$ such that the following conditions are satisfied.

(1) $\left|G_{a}\right| \leq x_{n-1}$.

(2) For each $a<\omega_{n}, A \cap G_{a}=A_{\lambda(a)}$ for some ordinal $\lambda(a)<\omega_{n}$.

(3) For each $a<\omega_{n},\left\langle A, G_{\alpha}\right\rangle / A=B_{\mu(a)}$ for some ordinal $\mu(a)<\omega_{n}$.

From the exactness of the sequence $G_{a} \cap A \longrightarrow G_{a} \rightarrow\left\langle G_{a}, A\right\rangle / A$, we observe that $G_{a}$ is free since conditions (1)-(3) imply that $G_{a}$ is the extension of a free group by a free group. In order to show that $G \in \mathcal{F}_{n}$, it suffices to prove that $G_{a+1} / G_{a} \in \mathcal{F}_{n-1}$ for each $a$. From the exact sequence

$$
\left\langle G_{a+1} \cap A, G_{a}\right\rangle / G_{a}>G_{a+1} / G_{a} \rightarrow G_{a+1} /\left\langle G_{a+1} \cap A, G_{a}\right\rangle
$$

and the isomorphisms

$$
\begin{gathered}
G_{a+1} /\left\langle G_{a+1} \cap A, G_{a}\right\rangle \cong\left(\left\langle A, G_{a+1}\right\rangle / A\right) /\left(\left\langle A, G_{a}\right\rangle / A\right) \\
\left\langle G_{a+1} \cap A, G_{a}\right\rangle / G_{a} \cong G_{a+1} \cap A / G_{a} \cap A,
\end{gathered}
$$

we obtain the exact sequence

$$
\dot{A}_{\lambda(a+1)} / A_{\lambda(a)} \longrightarrow G_{a+1} / G_{a} \rightarrow B_{\mu(a+1)^{/} B_{\mu(a)}} .
$$

Since $A_{\lambda(a+1)} / A_{\lambda(a)}$ and $B_{\mu(a+1)} / B_{\mu(a)}$ are contained in $\mathcal{F}_{n-1}$, the induction hypothesis $P(n-1)$ implies that $G_{a_{+} 1} / G_{\alpha} \in \mathcal{F}_{n-1}$ and that $G \in \mathcal{F}_{n}$. This completes the proof of the implication $P(n-1)+L(n) \Rightarrow P(n)$.

4. $T(n)+P(n-1)+C(n-1) \Rightarrow L(n)$. Let $G \in \mathcal{F}_{n}$. If $G$ is free, the conclusion of $L(n)$ is trivial. Thus we may assume, in view of $T(n)$, that $|G|=x_{n}$. Since $G \in \mathfrak{F}_{n}$, we know that $G$ has a representation as the union of a smooth chain $G_{0} \subseteq G_{1} \subseteq \cdots \subseteq G_{\alpha} \subseteq \cdots, \alpha<\mu$, of free groups $G_{\alpha}$, where $\mu \leq \omega_{n}$ and $G_{a+1} / G_{a} \epsilon$ 
$\mathcal{F}_{n-1}$ for each $\alpha$. If $\mu<\omega_{n}$, the restriction $\left|G_{a+1} / G_{a}\right| \leq x_{n-1}$ implies that $\left|G / G_{0}\right| \leq x_{n-1}$. Since $G_{0}$ is free, this implies that $G=F+H$ where $F$ is a summand of $G_{0}$ and $H$ has cardinality at most $x_{n-1}$. But $T(n)$ implies that $H$ is free and, consequently, $G$ is free. Thus without loss of generality we assume that $\mu=\omega_{n} \cdot$

For each ordinal $\alpha<\omega_{n}$, write $G_{a}=\Sigma_{i \in I(\alpha)}\left\langle x_{i}\right\rangle ;$ this is possible since $G_{a}$ is free. Now, we carefully choose a smooth chain $H_{0} \subseteq H_{1} \subseteq \cdots \subseteq H_{\lambda} \subseteq \cdots$, $\lambda<\omega_{n}$, of subgroups $H_{\lambda}$ of $G$ satisfying the following conditions.

(1) $\left|H_{\lambda}\right| \leq x_{n-1}$.

(2) $H_{\lambda} \subseteq G_{\lambda}$.

(3) $H_{\lambda}=\Sigma_{i \in J(\lambda)}\left\langle x_{i}\right\rangle$ if $\lambda$ is isolated, where $J(\lambda)$ is a subset of $I(\lambda)$.

(4) For each $\lambda<\omega_{n}, H_{\lambda} \cap G_{a}=\Sigma_{i \in J(a, \lambda)}\left\langle x_{i}\right\rangle$ if $\alpha<\lambda$, where $J(\alpha, \lambda)$ is a subset of $I(\alpha)$.

(5) For each $\lambda<\omega_{n}, G_{a+1} \subseteq\left\langle G_{a}, H_{\lambda}\right\rangle$ if $\alpha<\lambda$.

(6) $G=\bigcup_{\lambda<\omega_{n}} H_{\lambda}$.

Condition (1) implies that $H_{\lambda}$ is free. In order to establish $L(n)$, we need to prove that $H_{\beta} / H_{\alpha} \in \mathcal{F}_{n-1}$ whenever $\alpha<\beta<\omega_{n}$. First, we show that $H_{\lambda+1} / H_{\lambda} \in \mathcal{F}_{n-1}$ if $\lambda<\omega_{n} \cdot$ Observe that $G_{\lambda} / H_{\lambda}$ is free by condition (3) if $\lambda$ is isolated. Moreover, if $\lambda$ is a limit, then $G_{\lambda}=\bigcup_{a<\lambda}\left\langle G_{a}, H_{\lambda}\right\rangle$ since the chain of subgroups $G_{a}$ is smooth and since $H_{\lambda} \subseteq G_{\lambda}$. However, $\left\langle G_{a}, H_{\lambda}\right\rangle=\left\langle G_{0}, H_{\lambda}\right\rangle$ if $\alpha<\lambda$ by condition (5). Thus

$$
G_{\lambda} / H_{\lambda}=\left\langle G_{0}, H_{\lambda}\right\rangle / H_{\lambda} \cong G_{0} / G_{0} \cap H_{\lambda}
$$

is free by condition (3). We conclude that $G_{\lambda} / H_{\lambda}$ is free for all $\lambda<\omega_{n}$, and therefore $\left(H_{\lambda+1} \cap G_{\lambda}\right) / H_{\lambda}$ belongs to $\mathcal{F}_{n-1}$ since it is free and has cardinality not exceeding $x_{n-1}$. In view of $P(n-1), H_{\lambda+1} / H_{\lambda} \in \mathcal{F}_{n-1}$ if $H_{\lambda+1} /\left(H_{\lambda+1} \cap G_{\lambda}\right)$ belongs to $\mathcal{F}_{n-1}$. However, the equalities

$$
H_{\lambda+1} /\left(H_{\lambda+1} \cap G_{\lambda}\right) \cong\left\langle H_{\lambda+1}, G_{\lambda}\right\rangle / G_{\lambda}=G_{\lambda+1} / G_{\lambda}
$$

show that $H_{\lambda+1} /\left(H_{\lambda+1} \cap G_{\lambda}\right)$ is in $\mathcal{F}_{n-1}$. Since $H_{\lambda} / H_{\lambda+1} \cap G_{\lambda} \in \mathcal{F}_{n-1}$, we observe that simple applications of $P(n-1)$ and $C(n-1)$ show that $H_{\beta} / H_{\alpha} \in \mathcal{F}_{n-1}$ if $\alpha<\beta<\omega_{n}$ by induction on $\beta$.

5. $T(n)+P(n-1)+P(n)+L(n)+C(n-1) \Rightarrow C(n)$. Let $G$ be the union of the smooth chain

$$
G_{0} \subseteq G_{1} \subseteq \cdots \subseteq G_{a} \subseteq \cdots, \quad \alpha<\mu,
$$

of groups $G_{a} \in \mathcal{F}_{n}$, where $|\mu| \leq \aleph_{n}$ and $G_{a+1} / G_{a} \in \mathcal{F}_{n}$ for each $a<\mu$. Our purpose 
is to prove that $G$ is in $\mathfrak{F}_{n}$. If $G$ is free, then $G \in \mathcal{F}_{n}$ since the cardinality of $G$ does not exceed $x_{n}$. In particular, if $|G| \leq x_{n-1}$, then $G \in \mathcal{F}_{n}$ as a consequence of $T(n)$. Thus we shall assume that $|G|=\aleph_{n}$. The next point is that there is no loss of generality in assuming that $\mu \leq \omega_{n}$ for the following reason. If $C(n)$ is false and if $\mu$ is chosen as small as possible so that $G \notin \mathcal{F}_{n}$, then $P(n)$ and the choice of $\mu$ imply that the conditions on the chain of groups $G_{a}$ are retained by any smooth cofinal subchain. Since $\mu$ must be cofinal with $\omega_{i}$ for some $i \leq n$, it suffices to consider the case $\mu \leq \omega_{n}$. Therefore, we assume $\mu \leq \omega_{n}$ and distinguish the case $\mu<\omega_{n}$ from the case that $\mu=\omega_{n}$. However, a few more preliminaries are required yet.

For each $\lambda<\mu$, Lemma $L(n)$ gives a representation of $G_{\lambda}$ as the union of a smooth chain

$$
G_{0}(\lambda) \subseteq G_{1}(\lambda) \subseteq \cdots \subseteq G_{a}(\lambda) \subseteq \cdots, \quad a<\omega_{n},
$$

of free groups $G_{a}(\lambda)$ such that $\left|G_{a}(\lambda)\right| \leq x_{n-1}$ and $G_{\beta}(\lambda) / G_{a}(\lambda) \in \mathcal{F}_{n-1}$ if $\alpha<$ $\beta<\omega_{n}$. Likewise, $G_{\lambda+1} / G_{\lambda}$ has a representation as the union of a smooth chain

$$
G_{0}^{\lambda} \subseteq G_{1}^{\lambda} \subseteq \cdots \subseteq G_{a}^{\lambda} \subseteq \cdots, \quad a<\omega_{n},
$$

of free groups $G_{\alpha}^{\lambda}$ such that $\left|G_{a}^{\lambda}\right| \leq x_{n-1}$ and $G_{\beta}^{\lambda} / G_{a}^{\lambda} \in \mathcal{F}_{n-1}$ if $a<\beta<\omega_{n}$.

Case 1: $\mu=\omega_{n}$. For this case, the proof that $G \in \mathscr{F}_{n}$ is similar to a portion of the proof of the implication $T(n)+P(n-1)+C(n-1) \stackrel{n}{\Rightarrow} L(n)$, but the freeness of $G_{\lambda}$ has to be replaced here by the representation of $G_{\lambda}$ as the union of its free subgroups $G_{\alpha}(\lambda)$. We select a smooth chain

$$
H_{0} \subseteq H_{1} \subseteq \cdots \subseteq H_{\lambda} \subseteq \cdots, \quad \lambda<\omega_{n},
$$

of subgroups $H_{\lambda}$ of $G$ satisfying the following conditions.

(1) $\left|H_{\lambda}\right| \leq x_{n-1}$.

(2) $H_{\lambda} \subseteq G_{\lambda}$.

(3) If $\lambda$ is isolated, $H_{\lambda}=G_{\tau}(\lambda)$ for some $\tau$.

(4) For each $\lambda<\omega_{n}, H_{\lambda} \cap G_{a}=G_{\tau}(a)$ for some $r$ if $a<\lambda$.

(5) For each $\lambda<\omega_{n},\left\langle H_{\lambda} \cap G_{a+1}, G_{a}\right\rangle / G_{a}=G_{\tau}^{\alpha}$ for some $r$ if $a<\lambda$.

(6) $G=\bigcup_{\lambda<\omega_{n}} H_{\lambda}$.

It is not important (nor intended) that $\tau$ be the same in conditions (3)-(5), but it can be so selected if desired. It should be understood, of course, that $r$ depends on the choice of $\lambda$. Conditions (1) and (2), together with $T(n)$, imply that $H_{\lambda}$ is free. Thus we wish to prove that $H_{\lambda+1} / H_{\lambda} \in \mathcal{F}_{n-1}$ in order to verify that $G \in \mathcal{F}_{n}$. According to $P(n-1)$, it suffices to prove that $\left(H_{\lambda_{+}} \cap G_{\lambda}\right) / H_{\lambda} \in \mathcal{F}_{n-1}$ because we have the exact sequence 


$$
\left(H_{\lambda+1} \cap G_{\lambda}\right) / H_{\lambda} \longrightarrow H_{\lambda+1} / H_{\lambda} \rightarrow H_{\lambda+1} /\left(H_{\lambda+1} \cap G_{\lambda}\right)
$$

and $H_{\lambda+1} /\left(H_{\lambda+1} \cap G_{\lambda}\right) \cong\left\langle H_{\lambda+1}, G_{\lambda}\right\rangle / G_{\lambda}$ obviously belongs to $\mathcal{F}_{n-1}$ since it has cardinality at most $x_{n-1}$ and, therefore, is isomorphic to a free subgroup of $G_{\lambda+1} / G_{\lambda}$ by $T(n)$. In order to verify that $\left(H_{\lambda+1} \cap G_{\lambda}\right) / H_{\lambda}$ is in $\mathcal{F}_{n-1}$, we first consider the case that $\lambda$ is isolated. In this case, $\left(H_{\lambda_{+}+1} \cap G_{\lambda}\right) / H_{\lambda}=G_{\tau}(\lambda) / G_{\sigma}(\lambda)$ for appropriate $\sigma$ and $r \geq \sigma$. Thus $\left(H_{\lambda+1} \cap G_{\lambda}\right) / H_{\lambda} \in \mathcal{F}_{n-1}$. Now suppose that $\lambda$ is a limit ordinal. In this case, $\left(H_{\lambda+1} \cap G_{\lambda}\right) / H_{\lambda}$ is the union of the smooth chain

$$
\left\langle H_{\lambda+1} \cap G_{0}, H_{\lambda}\right\rangle / H_{\lambda} \subseteq \cdots \subseteq\left\langle H_{\lambda+1} \cap G_{\alpha}, H_{\lambda}\right\rangle / H_{\lambda} \subseteq \cdots, \quad a<\lambda_{0}
$$

Observe that

$$
\left\langle H_{\lambda+1} \cap G_{\alpha}, H_{\lambda}\right\rangle / H_{\lambda} \cong\left(H_{\lambda+1} \cap G_{\alpha}\right) /\left(H_{\lambda} \cap G_{\alpha}\right)=G_{\tau}(a) / G_{\sigma}(a)
$$

belongs to $\mathcal{F}_{n-1}$. Furthermore,

$$
\left\langle H_{\lambda+1} \cap G_{a+1}, H_{\lambda}\right\rangle /\left\langle H_{\lambda+1} \cap G_{a}, H_{\lambda}\right\rangle \cong\left\langle H_{\lambda+1} \cap G_{a+1}, G_{a}\right\rangle\left\langle\left(H_{\lambda} \cap G_{a+1}, G_{a}\right\rangle,\right.
$$

so

$$
\left(\left\langle H_{\lambda+1} \cap G_{a+1}, H_{\lambda}\right\rangle / H_{\lambda}\right) /\left(\left\langle H_{\lambda+1} \cap G_{a}, H_{\lambda}\right\rangle / H_{\lambda}\right) \cong G_{\tau}^{a} / G_{\sigma}^{a}
$$

is in $\mathcal{F}_{n-1}$. We conclude that $\left(H_{\lambda+1} \cap G_{\lambda}\right) / H_{\lambda}$ belongs to $\mathcal{F}_{n-1}$ by $C(n-1)$. This completes the proof for the case $\mu=\omega_{n}$, but we still have to deal with the alternative.

Case 2: $\mu<\omega_{n}$. Again, we can choose a smooth chain

$$
H_{0} \subseteq H_{1} \subseteq \cdots \subseteq H_{\lambda} \subseteq \cdots, \quad \lambda<\omega_{n},
$$

of subgroups $H_{\lambda}$ of $G$ satisfying the following conditions.

(1) $\left|H_{\lambda}\right| \leq x_{n-1}$.

(4') For each $\lambda<\omega_{n}$ and $\alpha<\mu, H_{\lambda} \cap G_{a}=G_{\tau}(\alpha)$ for some $r$.

(5') For each $\lambda<\omega_{n}$ and $\alpha<\mu,\left\langle H_{\lambda} \cap G_{a+1}, G_{a}\right\rangle / G_{a}=G_{\tau}^{a}$ for some $r$.

(6) $G=\bigcup_{\lambda<\omega_{n}} H_{\lambda}$.

In order to prove that $G \in \mathcal{F}_{n}$, we need to prove that $H_{\lambda}$ is free and that $H_{\lambda+1} / H_{\lambda} \epsilon$ $\mathcal{F}_{n-1}$. Although Theorem $T(n)$ does not apply directly in the present case to yield the freeness of $H_{\lambda}$, we observe that $H_{\lambda}$ is the union of the smooth chain

$$
H_{\lambda} \cap G_{0} \subseteq H_{\lambda} \cap G_{1} \subseteq \cdots \subseteq H_{\lambda} \cap G_{a} \subseteq \cdots, \quad \alpha<\mu,
$$

of free groups. Moreover, $\left(H_{\lambda} \cap G_{a+1}\right) /\left(H_{\lambda} \cap G_{\alpha}\right)$ is isomorphic to a subgroup of $G_{a+1} / G_{a}$, and therefore is free by $T(n)$. Thus $H_{\lambda}$ is free. In order to show that $H_{\lambda+1} / H_{\lambda} \in \mathcal{F}_{n-1}$, we represent $H_{\lambda+1} / H_{\lambda}$ as the union of the smooth chain

$$
\left\langle H_{\lambda+1} \cap G_{0}, H_{\lambda}\right\rangle / H_{\lambda} \subseteq \cdots \subseteq\left\langle H_{\lambda+1} \cap G_{a}, H_{\lambda}\right\rangle / H_{\lambda} \subseteq \cdots, \quad a<\mu .
$$


Since $\left\langle H_{\lambda+1} \cap G_{a}, H_{\lambda}\right\rangle / H_{\lambda} \simeq G_{\tau}(a) / G_{\sigma}(a)$ belongs to $\mathcal{F}_{n-1}$ and since $|\mu| \leq x_{n-1}$ it suffices by $C(n-1)$ to prove that

$$
\left\langle H_{\lambda+1} \cap G_{a+1}, H_{\lambda}\right\rangle /\left\langle H_{\lambda+1} \cap G_{a}, H_{\lambda}\right\rangle
$$

is in $\mathcal{F}_{n-1}$. As in the preceding case, the latter is is omorphic to $G_{\tau}^{\alpha} / G_{\sigma}^{a}$. Thus the proof is complete.

6. $T(n)+L(n)+P(n)+C(n) \Rightarrow T(n+1)$. Let $G \in \mathcal{F}_{n+1}$ and let $H$ be a subgroup of $G$ of cardinality not exceeding $x_{n}$. Since $G \in \mathcal{F}_{n+1}, G$ is the union of a smooth chain

$$
G_{0} \subseteq G_{1} \subseteq \cdots \subseteq G_{a} \subseteq \cdots, \quad a<\mu,
$$

of free groups $G_{a}$, where $G_{a+1} / G_{a} \in \mathcal{F}_{n}$ and $\mu \leq \omega_{n+1}$. If $H \subseteq G_{a}$, we are finished. Assume not. Since

$$
H \cap G_{0} \subseteq H \cap G_{1} \subseteq \cdots \subseteq H \cap G_{a} \subseteq \cdots
$$

ascends to $H$, upon eliminating redundancies, we see that $H$ is the union of a smooth chain

$$
H_{0} \subseteq H_{1} \subseteq \cdots \subseteq H_{a} \subseteq \cdots, \quad a<\lambda,
$$

of free groups $H_{a}$, where $H_{a+1} / H_{a} \in \mathcal{F}_{n}$ and $|\lambda| \leq x_{n}$. By Corollary $C(n), H \in \mathcal{F}_{n}$. If $|H| \leq x_{n-1}$, then $H$ is free by $T(n)$. Thus we may assume that $|H|=x_{n}$. Furthermore, since $\lambda$ is cofinal with an ordinal not exceeding $\omega_{n}$, by $P(n)$ and $C(n)$ there is no loss of generality in assuming that $\lambda \leq \omega_{n}$. $\Lambda s$ before, we need to distinguish the case $\lambda=\omega_{n}$ from the case where $|\lambda| \leq x_{n-1}$. The basic design will fit closely that fashioned in preceding proofs. For each $\gamma<\lambda$, Lemma $L(n)$ yields a representation of $H_{\gamma+1} / H_{\gamma}$ as the union of a smooth chain $H_{0}^{\gamma} \mathrm{C}$ $H_{1}^{\gamma} \subseteq \cdots \subseteq H_{a}^{\gamma} \subseteq \cdots, a<\omega_{n}$, of free groups $H_{a}^{\gamma}$ such that $\left|H_{a}^{\gamma}\right| \leq x_{n-1}$ and $H_{\beta}^{\gamma} / H_{a}^{\gamma} \in \mathcal{F}_{n-1}$ if $a<\beta<\omega_{n}$. In the present situation, $H_{\gamma}$ not only has such a representation but in fact is free. Thus write $H_{\gamma}=\Sigma_{i \in I(\gamma)}\left\langle x_{i}\right\rangle$. We choose a smooth chain

$$
F_{0} \subseteq F_{1} \subseteq \cdots \subseteq F_{\alpha} \subseteq \cdots, \quad \alpha<\omega_{n},
$$

of subgroups $F_{a}$ of $H$ so that the following conditions are satisfied.

Case 1: $\lambda=\omega_{n}$.

(1) $\left|F_{a}\right| \leq x_{n-1}$.

(2) $F_{a} \subseteq H_{a}$.

(3) If $\alpha$ is isolated, $F_{a}=\Sigma_{i \in J(a)}\left\langle x_{i}\right\rangle$ where $J(\alpha)$ is a subset of $I(\alpha)$.

(4) For each $a<\omega_{n}, F_{a} \cap H_{\gamma}=\Sigma_{i \in J(\gamma, \alpha)}\left\langle x_{i}\right\rangle$ whenever $\gamma<a$, where $J(\gamma, a)$ is a subset of $I(\gamma)$.

(5) For each $a<\omega_{n},\left\langle F_{a} \cap H_{\gamma+1}, H_{\gamma}\right\rangle / H_{\gamma}=H_{\tau}^{\gamma}$ for some $r$ if $a>\gamma$.

(6) $H=\bigcup_{a<\omega_{n}} F_{a^{*}}$ 
Case 2: $\lambda<\omega_{n}$. In this case, we retain conditions (1) and (6), eliminate conditions (2) and (3), and strengthen conditions (4) and (5) as follows.

(4') For each $\alpha<\omega_{n}$ and each $\gamma<\lambda, F_{a} \cap H_{\gamma}=\Sigma_{i \in J(\gamma, a)}\left\langle x_{i}\right\rangle$ with $J(\gamma, \alpha) \subseteq I(\gamma)$.

$\left(5^{\prime}\right)$ For each $a<\omega_{n}$ and each $\gamma<\lambda,\left\langle F_{\alpha} \cap H_{\gamma+1}, H_{\gamma}\right\rangle / H_{\gamma}=H_{r}^{\gamma}$ for some $r$.

Obviously $F_{a}$ is free according to $T(n)$ since $H \in \mathcal{F}_{n}$. The verification that $F_{a+1} / F_{a}$ is free will show that $H$ is free in view of condition (6), and the proof will be complete. Moreover, it is enough to show that $F_{a+1} / F_{\alpha} \in . \mathcal{F}_{n}$ since we can apply $T(n)$. In Case 1 , we have the exact sequence

$$
\left(F_{a+1} \cap H_{\alpha}\right) / F_{a} \longrightarrow F_{a+1} / F_{a} \rightarrow\left\langle F_{a+1}, H_{a}\right\rangle / H_{\alpha}
$$

Since $F_{a} \subseteq H_{a}$ and since $H_{a+1} / H_{a} \in \mathcal{F}_{n}$, we observe that it suffices to prove that $\left(F_{a+1} \cap H_{\alpha}\right) / F_{a} \in \mathcal{F}_{n}$ because we can apply $P(n)$. If $\alpha$ is isolated, this is trivial because $H_{\alpha} / F_{a}$ is free according to condition (3). Thus assume that $\alpha$ is a limit, and represent $\left(F_{a_{+}} \cap H_{a}\right) / F_{a}$ as the union of the smooth chain

$$
\left\langle F_{a+1} \cap H_{0}, F_{a}\right\rangle / F_{a} \subseteq \cdots \subseteq\left\langle F_{a+1} \cap H_{\gamma}, F_{a}\right\rangle / F_{a} \subseteq \cdots, \quad \gamma<\alpha,
$$

of free groups

$$
\left\langle F_{a+1} \cap H_{\gamma}, F_{a}\right\rangle / F_{a} \cong\left(F_{a+1} \cap H_{\gamma}\right) /\left(F_{\alpha} \cap H_{\gamma}\right)=\sum_{i \in J(\gamma, a+1)-J(\gamma, a)}\left\langle x_{i}\right\rangle .
$$

The isomorphisms

$$
\begin{aligned}
& \left\langle F_{a+1} \cap H_{\gamma+1}, F_{a}\right\rangle /\left\langle F_{a+1} \cap H_{\gamma}, F_{a}\right\rangle \\
& \approx\left\langle F_{a+1} \cap H_{\gamma+1}, H_{\gamma}\right\rangle /\left\langle F_{a} \cap H_{\gamma+1}, H_{\gamma}\right\rangle=H_{\tau}^{\gamma} / H_{\sigma}^{\gamma}
\end{aligned}
$$

reveal that $\left\langle F_{a+1} \cap H_{a}\right\rangle / F_{a}$ and, therefore, $F_{a+1} / F_{a}$ belong to $\mathcal{F}_{n}$ since $H_{\tau}^{\gamma} / H_{\sigma}^{\gamma} \in \mathcal{F}_{n-1}$ and since $\alpha \leq \omega_{n}$.

If $\lambda<\omega_{n}$, we represent $F_{a_{+1}} / F_{a}$ as the union of the smooth chain

$$
\left\langle F_{a+1} \cap H_{0}, F_{a}\right\rangle / F_{a} \subseteq \cdots \subseteq\left\langle F_{a+1} \cap H_{\gamma}, F_{a}\right\rangle / F_{a} \subseteq \cdots, \quad \gamma<\lambda_{b}
$$

of free groups $\left\langle F_{a+1} \cap H_{\gamma}, F_{\alpha}\right\rangle / F_{a}$. All that is required now is to prove that $\left\langle F_{a+1} \cap H_{\gamma+1}, F_{a}\right\rangle /\left\langle F_{a+1} \cap H_{\gamma}, F_{a}\right\rangle$ is in $\mathcal{F}_{n-1}$. However, the proof of this is similar to what has gone before, and we suppress further details.

7. The general proofs. In the previous sections we have established the following implications

$$
\begin{aligned}
& P(n-1)+L(n) \Rightarrow P(n), \\
& T(n)+P(n-1)+C(n-1) \Rightarrow L(n), \\
& T(n)+P(n-1)+P(n)+L(n)+C(n-1) \Rightarrow C(n), \\
& T(n)+L(n)+P(n)+C(n) \Rightarrow T(n+1)
\end{aligned}
$$


for each positive integer $n$. Since $\mathcal{F}_{0}$ is the class of all countable torsion-free groups, both the proposition and the corollary are trivially true for $n=0$. Moreover, the validity of the theorem for $n=1$ follows immediately from Pontryagin's theorem. Thus we start with $P(0), C(0)$ and $T(1)$. Inductively, everything now follows from the preceding implications. Therefore, the lemma, proposition, corollary, and main theorem are proved in general.

Referring to the groups belonging to the class $\mathcal{F}_{n}$ as $\beth_{n}$-free groups, we have proved the following for every positive integer $n$.

(1) A subgroup of a $\beth_{n}$-free group is $\beth_{n}$-free.

(2) An extension of a $J_{n}$-free group by an $\beth_{n}$-free group is $J_{n}$-free.

(3) Any $J_{n}$-free group is $x_{n}$-free.

Except for the distinction as main theorem which we have given it, the result that $ב_{n}$-free groups are $x_{n}$-free appears unpretentious. Nonetheless it contains, in essence, the following uniqueness theorem, which is of considerable interest and generalizes results in [5] and [6].

Theorem 2. If $G$ is the union of a smooth chain $G_{0} \subseteq G_{1} \subseteq \cdots \subseteq G_{a} \subseteq \cdots$ of length not exceeding $\boldsymbol{X}_{n}(n<\omega)$ of free groups $G_{a}$, then $G$ is free provided that $G_{a+1} / G_{\alpha}$ is $2_{n}$-free for each $\alpha$.

Proof. Observe that $\left|G / G_{0}\right| \leq \star_{n}$. Since $G_{0}$ is free, we can write $G=$ $H_{0}+A$, where $H_{0}$ is a direct summand of $G_{0}$ and $|A| \leq \aleph_{n}$. By hypothesis, $G$ is $z_{n+1}$-free. Thus $G$ is $x_{n+1}-$ free, and $A$ is free. Therefore, $G$ is free since both $H_{0}$ and $A$ are free.

8. The existence theorem. In this section, the uniqueness theorem is utilized heavily in establishing an existence theorem for nonfree groups that are $\beth_{n}$-free.

Theorem 3. For every nonnegative integer $n$, there exist $\nu_{n}$-free groups that are not free.

Proof. The proof is by induction on $n$, and the proof is trivial for $n=0$ since all countable torsion-free groups are $2_{0}$-free. Suppose that $n>0$ and that the theorem is valid for all nonnegative integers less than or equal to $n$. Assume that for an ordinal $\mu$ of cardinality at most $x_{n}$, we have developed a smooth chain $G_{0} \subseteq G_{1} \subseteq \cdots \subseteq G_{a} \subseteq \cdots, a<\mu$, of free groups $G_{a}$ of cardinality $\aleph_{n}$ so that $G_{a_{+}+1} / G_{a}$ is a nonfree $J_{n}$-free group whenever $\alpha+1<\mu$. If $\mu$ is isolated we choose $G_{\mu}$ to be any free group containing $G_{\mu-1}$ so that $G_{\mu-1}>G_{\mu} \rightarrow H_{\mu}$ is exact, where $H_{\mu}$ is any $\beth_{n}$-free group that is not free; the existence of a group meeting the requirements of $H_{\mu}$ is guaranteed by the induction hypothesis. Since $H_{\mu}$ must have cardinality $\aleph_{n}$, there is a free group $G_{\mu}$ of cardinality $\aleph_{n}$ with an 
epimorphism $G_{\mu} \rightarrow H_{\mu}$. Obviously, the kernel of this epimorphism must have cardinality $x_{n}$, for otherwise $H_{\mu}$ could not be $x_{n}$-free without being free. Therefore, the kernel can be identified with $G_{\mu-1}$, and we have the desired exact sequence $G_{\mu-1} \gg G_{\mu} \rightarrow H_{\mu}$. If $\mu$ is a limit, we define $G_{\mu}=\bigcup_{\alpha<\mu} G_{a}$. Then $\left|G_{\mu}\right|=x_{n}$ since $|\mu| \leq x_{n}$ and since $\left|G_{\alpha}\right|=x_{n}$ for each $\alpha<\mu$. In this case, $G_{\mu}$ is free by Theorem 2 .

We have verified the existence of a smooth chain

$$
G_{0} \subseteq G_{1} \subseteq \cdots \subseteq G_{a} \subseteq \cdots, \quad a<\omega_{n+1},
$$

of free groups $G_{\alpha}$ of cardinality $x_{n}$ such that $G_{a+1} / G_{a}$ is $J_{n}$-free but not free for each $a<\omega_{n+1}$. Let $G=\bigcup_{a<\omega_{n+1}} G_{a}$. Clearly, $G$ is $\beth_{n+1}$-free. However, $G$ is not free for the following simple reason. If $G$ were free, then $G_{\alpha}$ would have to be a direct summand of $G$ for some $\alpha$ (by a simple application of the back-andforth procedure used implicitly throughout this paper). Since $G_{a_{+}+1} / G_{a}$ is not free, there is no way that $G_{a}$ can be a direct summand of $G$ for any $a$. Hence we conclude that $G$ is not free, and the theorem is proved.

Corollary 1. For each positive integer $n$, there exists a group of cardinality $x_{n}$ that is not free but is $x_{n}$-free.

9. The proof of a conjecture in infinitary logic. The following conjecture was made in [6].

Conjecture. If $\kappa=x_{n}$ for any positive integer $n$, the class of free groups is not definable in $L_{\infty K^{\circ}}$

For a discussion of the language $L_{\infty K^{\prime}}$, we refer to [1] and [2]. However, all that is needed for our purpose is the following result from [1]. $\Lambda$ group $G$ is equivalent in $L_{\infty K}$ to a free group if and only if $G$ has the property that every subgroup $H$ generated by fewer than $\kappa$ elements is contained in a free subgroup $F$ of $G$ that splits out of $\langle F, A\rangle$ whenever $A$ is a subgroup of $G$ generated by fewer than $\kappa$ elements.

Proof of conjecture. Let $G$ be a nonfree group that is $J_{n+1}$-free. Since $G$ is $z_{n+1}$-free, $G$ is the union of a smooth chain $G_{0} \subseteq G_{1} \subseteq \cdots \subseteq G_{a} \subseteq \cdots, a<\omega_{n+1}$, of free groups $G_{a}$ such that $G_{a+1} / G_{a}$ is $\beth_{n}$-free. Let $H$ be any subgroup of $G$ having cardinality not exceeding $x_{n-1}$. Then $H \subseteq G_{a}$ for some $\alpha$. Moreover, if $G_{a} \subseteq K \subseteq G$ and if $\left|K / G_{a}\right| \leq x_{n-1}$, then $K \subseteq G_{\beta}$ for some $\beta$. We know that $G_{\beta} / G_{a}$ is $\beth_{n}$-free by the proposition and corollary. Thus $G_{\beta} / G_{\alpha}$ is $x_{n}$-free, which implies that $K / G_{a}$ is free. Hence $G_{a}$ splits out of $K$. This completes the proof of the conjecture. 


\section{REFERENCES}

1. P. Eklof, Infinitary equivalence of abelian groups: with counterexamples to Scott's theorem in uncountable cardinals (preprint).

2. S. Feferman, Infinitary properties, local functors, and systems of ordinal functions, Conference in Mathematical Logic (London, 1970), Springer-Verlag, Berlin, 1972.

3. L. Fuchs, Infinite abelian groups. Vol. I, Pure and Appl. Math., vol. 36, Academic Press, New York, 1970. MR 41 \#333.

4. P. Griffith, $X_{n}-$ free abelian groups, Aarhus University preprint series, 1971/72.

5. P. Hill, On the splitting of modules and abelian groups, Canad. J. Math. 26 (1974), 68-77.

6. - New criteria for freeness in abelian groups, Trans, Amer. Math. Soc. 182 (1973), 201-209.

DEPARTMENT OF MATHEMATICS, FLORIDA STATE UNIVERSITY, TALLAHASSEE, FLORIDA 32306 